\title{
Palbociclib: First CDK4/6 Inhibitor in Clinical Practice for the Treatment of Advanced HR-Positive Breast Cancer
}

\author{
Johannes Ettl \\ Department of Obstetrics and Gynecology, Klinikum rechts der Isar, Technische Universität München, Munich, Germany
}

Keywords

Advanced breast cancer - Palbociclib - CDK4/6 .

Endocrine therapy · Cell cycle

\section{Summary}

Palbociclib is the first inhibitor of the cyclin-dependent kinases (CDK) 4 and 6 to be introduced into clinical practice. Preclinical investigations led to its clinical development in advanced hormone receptor (HR)-positive breast cancer. To date, 2 significant clinical trials have been fully published. In this article, the results of these trials and their clinical relevance for the management of HRpositive advanced breast cancer are discussed.

(C) 2016 S. Karger GmbH, Freiburg

\section{Introduction}

In February 2015, the U.S. Food and Drug Administration (FDA) approved palbociclib (Ibrance ${ }^{\circledR}$, Pfizer Deutschland GmbH, Berlin, Germany) for the treatment of postmenopausal women with estrogen receptor (ER)-positive, human epidermal growth factor receptor 2 (HER2)-negative advanced breast cancer as firstline therapy in combination with letrozole. With the development of palbociclib, a new therapy target - the cyclin-dependent kinases (CDK) 4 and 6 - has been introduced into the therapy of breast cancer. With the results of the phase III trial PALOMA-3 being already published [1] and first PALOMA-2 results being expected later this year, the application for approval of the drug has been submitted to the European Medicines Agency. The following article gives a condensed overview of the current role of palbociclib in the therapy of ER-positive breast cancer.

\section{Mechanism of Action}

The transition from the G1 to the S phase of the cell cycle has been identified as a therapy target. CDKs, cyclins, and the retinoblastoma protein $(\mathrm{Rb})$ play a crucial role in cell cycle progression [2]. Phosphorylation of $\mathrm{Rb}$ in the early $\mathrm{G} 1$ phase is a prerequisite for proliferation. $\mathrm{Rb}$ phosphorylation is influenced by CDKs, together with mitogenic stimuli by other upstream receptor tyrosine kinases. CDK4/6 inhibition by palbociclib in combination with anti-estrogens causes decreased $\mathrm{Rb}$ phosphorylation, leading to growth arrest by blocking the cell cycle [3]. Thus, palbociclib is able to enhance the antiproliferative effect of anti-estrogens.

\section{Preclinical Data}

In a preclinical study of 47 molecularly classified breast cancer cell lines, Finn et al. [4] demonstrated that palbociclib is able to inhibit $\mathrm{Rb}$ phosphorylation and cause growth inhibition. Luminal cell lines, representing the ER-positive subtype, and also ER-positive/HER2-positive cells were most sensitive to palbociclib. Palbociclib acted synergistically with tamoxifen in these cell lines. High expression levels of Rb and cyclin D1 and low expression of p16 were identified as predictors of response to palbociclib.

\section{Clinical Data}

In a phase I dose escalation study, palbociclib was examined in a 3-weeks-on / 1-week-off schedule in 41 patients with advanced malignancies [5]. The recommended phase II dose was determined to be $125 \mathrm{mg}$ once daily. As dose-limiting toxicity, reversible neutropenia was identified. Grade 3 hematologic toxicities included neutropenia (12\%) and anemia (7\%). Non-hematologic toxicity

\section{KARGER \\ Fax +497614520714

\section{() 2016 S. Karger GmbH, Freiburg}

$1661-3791 / 16 / 0113-0174 \$ 39.50 / 0$ 


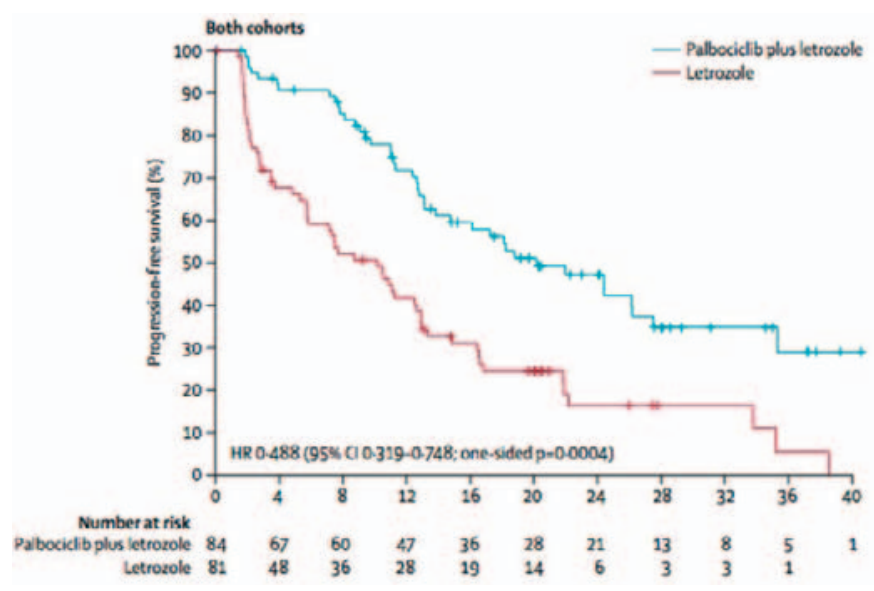

Fig. 1. Progression-free survival (intention-to-treat population) from the PALOMA-1 study [6].

was mild and included fatigue, nausea, and diarrhea. There was a clear signal for clinical activity of the drug: $35 \%$ of patients experienced stable disease as best response, including $6(16 \%)$ patients who received 10 or more cycles of therapy.

In the open-label phase II PALOMA-1 trial, 165 postmenopausal patients with advanced ER-positive and HER2-negative breast cancer, who had not received any systemic treatment for advanced disease, were randomized to $2.5 \mathrm{mg}$ letrozole either alone or in combination with $125 \mathrm{mg}$ palbociclib 3 weeks on / 1 week off [6]. The study comprised 2 cohorts: In cohort $1(n=66)$, patients were randomized without selection for potential biomarkers, whereas patients in cohort $2(n=99)$ were required to be positive for either amplification of cyclin D1 or loss of p16 or both. In the whole study population (cohorts 1 and 2), evaluation of the primary endpoint progression-free survival (PFS) showed a significant difference between the treatment arms: Median PFS was 10.2 months (95\% confidence interval (CI) 5.7-12.6) for the letrozole alone group and 20.2 months (95\% CI 13.8-27.5) for the palbociclib plus letrozole group (hazard ratio (HR) 0.488, 95\% CI 0.319-0.748; 1 -sided $\mathrm{p}=0.0004)$. Median follow-up was 29.6 months (95\% CI 27.9-36.0) for the palbociclib plus letrozole group and 27.9 months (25.5-31.1) for the letrozole alone group (fig. 1). This improvement in PFS with palbociclib was independent of the presence of the potential biomarkers cyclin D1 amplification or p16 loss, meaning that these 2 markers are not predictive of response to palbociclib. Subgroup analyses showed that patients derived benefit from palbociclib independent of site of metastasis (visceral vs. nonvisceral) [7], age ( $<65$ years vs. $\geq 65$ years) [8], and prior systemic therapies [9]. The analysis of the secondary endpoint overall survival (OS) did not show a significant difference between the 2 groups: Median OS was 37.5 months in the palbociclib plus letrozole group and 33.3 months in the letrozole alone group (HR 0.813, 95\% CI 0.492-1.345; $\mathrm{p}=0.42$ ).

The most common adverse event was neutropenia. Grade 3-4 neutropenia was reported in 45 (54\%) of 83 patients in the palbociclib plus letrozole group versus 1 (1\%) of 77 patients in the letrozole alone group. Neutropenia was not of any clinical relevance for the patients: No cases of febrile neutropenia or neutropenia-related infections were reported during the study. Fatigue was also more common in the palbociclib plus letrozole arm (4 vs. $1 \%$ for grades 3-4).

To date, 1 phase III study with palbociclib has been fully published [1]: PALOMA-3 was a randomized, double-blind, two-arm study investigating the combination of fulvestrant and palbociclib in pre- and postmenopausal patients with advanced ER-positive, HER2-negative breast cancer that had relapsed or progressed during prior endocrine therapy. Of the 521 randomized patients, 72 (21\%) were premenopausal or perimenopausal and, in addition to fulvestrant, received goserelin for ovarian suppression. The primary endpoint of the study was PFS. Secondary endpoints included OS, objective response, clinical benefit rate, patient-reported outcomes, and safety. Adding palbociclib to endocrine therapy with fulvestrant led to a significant and clinically meaningful improvement in median PFS from 3.8 months (95\% CI 3.5-5.5) to 9.2 months (95\% CI 7.5-not estimable), the HR for disease progression or death being 0.42 (95\% CI $0.32-0.56$; $\mathrm{p}<0.001)$. Subgroup analyses revealed that the benefit of palbociclib is irrespective of age, site of metastatic disease, prior chemotherapy, and menopausal status. In particular the HR for disease progression or death was similar in pre- or perimenopausal versus postmenopausal patients. This is of great importance, since the amount of data existing for endocrine-based treatment options in premenopausal metastatic breast cancer is currently very limited. OS results for PALOMA-3 have been immature so far.

As in PALOMA-1, palbociclib was well tolerated in PALOMA-3: The most common grade 3 or 4 adverse events were neutropenia ( 62.0 vs. $0.6 \%$ in the placebo/fulvestrant groups, respectively), anemia ( 2.6 vs. $1.7 \%$ ), thrombocytopenia ( 2.3 vs. $0 \%$ ), and fatigue ( 2.0 vs. $1.2 \%$ ). Febrile neutropenia was reported in only 2 $(0.6 \%)$ of the palbociclib-treated patients. The rate of discontinuation due to adverse events was $2.6 \%$ with palbociclib and $1.7 \%$ with placebo.

The difference between PFS rates in PALOMA-1 and PALOMA-3 might be due to the fact that both studies recruited different patient populations (endocrine-sensitive vs. endocrine-resistant disease, first-line vs. second/third-line therapy).

\section{Clinical Relevance of Palbociclib in the Treatment of Metastatic Breast Cancer}

The main goal of treatment in metastatic breast cancer is to prevent disease progression while maintaining physical efficiency and quality of life during the course of the chronic disease. This is why patients with endocrine-sensitive metastatic breast cancer should preferably receive endocrine therapy unless there is visceral crisis [10]. When introducing new therapies into endocrine treatment strategies, it is of great importance that the possible gain in efficacy does not happen at the expense of treatment tolerability and quality of life. Palbociclib has been shown to double treatment efficacy of letrozole and fulvestrant with a restricted and well-manageable 


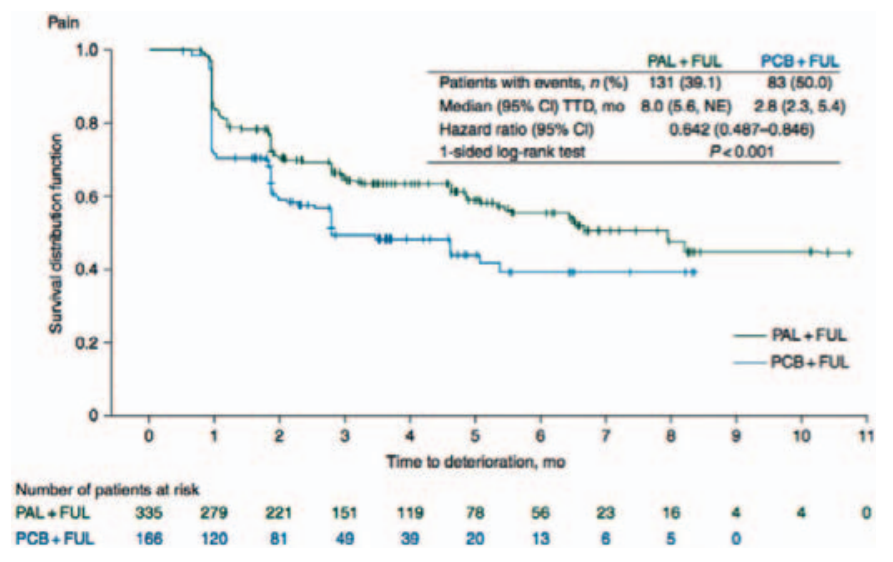

Fig. 2. Time to deterioration (TTD) in pain in the analyses of patient reported outcomes (PRO) from the PALOMA-3 trial [11].

NE, not estimable; PAL, palbociclib; FUL, fulvestrant; PCB, placebo.

toxicity profile. The $2.6 \%$ rate of adverse event-related therapy discontinuation in PALOMA-3 [1] has been unprecedented in other comparable phase III trials combining endocrine therapy with biologicals. The neutropenia associated with palbociclib is different from chemotherapy-induced neutropenia: It does not go along with a significantly increased rate of febrile neutropenia or clinically relevant infections. It might be interpreted as the expression of a cytostatic (cell cycle-blocking) rather than a cytotoxic (cell-destroying) effect. Since its approval by the FDA in the USA and an estimated number of 20,000 patients having been treated with palbociclib, no recent safety concerns have been reported.

A thorough analysis of the patient-reported outcomes from the PALOMA-3 trial has recently been published [11]. It was shown that the overall global quality of life scores of palbociclib-treated patients were significantly higher than the scores of the patients in the control group (66.1, 95\% CI 64.5-67.7 vs. $63.0,95 \%$ CI $60.6-$ $65.3 ; \mathrm{p}=0.0313$ ). A significantly greater delay in deterioration of quality of life was observed in the palbociclib plus fulvestrant group versus the control arm (median not reached; HR 0.641, 95\% CI $0.451-0.910 ; \mathrm{p}=0.0065)$. Moreover, median estimated time to deterioration (TTD) of pain was 8 months (95\% CI 5.6-not estimable) in the palbociclib plus fulvestrant group compared with 2.8 months (95\% CI 2.3-5.4) in the placebo plus fulvestrant group. Treatment with palbociclib significantly delayed TTD of pain symptoms versus placebo plus fulvestrant (HR 0.642, 95\% CI 0.487-0.846; $\mathrm{p}<0.001$ ) (fig. 2).

\section{Conclusion}

Palbociclib is the first-in-class CDK4/6 inhibitor to be introduced into clinical practice. Present clinical data characterize it as a highly effective, well-tolerated biological to be combined with letrozole or fulvestrant in hormone receptor (HR)-positive, locally advanced, and/or metastatic breast cancer. The upcoming results of the PALOMA-2 trial are eagerly awaited. PALOMA-2 is a randomized, double-blind, phase III trial of palbociclib with letrozole compared with letrozole alone as first-line therapy in postmenopausal women with ER-positive/HER2-negative advanced breast cancer. The trial was designed to confirm the phase II results in the first-line setting, and thus will further clarify the role of palbociclib in treatment strategies for HR-positive advanced breast cancer.

\section{Disclosure Statement}

The author received honoraria from Amgen, AstraZeneca, Celgene, gsk, Eisai, Novartis, Pfizer, Pierre Fabre, and Roche.

\section{References}

1 Turner NC, Ro J, Andre F, et al.: Palbociclib in hormone-receptor-positive advanced breast cancer. $\mathrm{N}$ Engl J Med 2015;373:209-219.

12 Fry DW, Harvey PJ, Keller PR, et al.: Specific inhibition of cyclin-dependent kinase 4/6 by PD 0332991 and associated antitumor activity in human tumor xenografts. Mol Cancer Ther 2004;3:1427-1438.

3 Caldon CE, Daly RJ, Sutherland RL, et al.: Cell cycle control in breast cancer cells. J Cell Biochem 2006;97: 261-274.

4 Finn RS, Dering J, Conklin D, et al.: PD 0332991, a selective cyclin D kinase 4/6 inhibitor, preferentially inhibits proliferation of luminal estrogen receptor-positive human breast cancer cell lines in vitro. Breast Cancer Res 2009;11:R77.

5 Flaherty KT, Lorusso PM, Demichele A, et al.: Phase I, dose-escalation trial of the oral cyclin-dependent kinase 4/6 inhibitor PD 0332991, administered using a 21-day schedule in patients with advanced cancer. Clin Cancer Res 2012;18:568-576.
6 Finn RS, Crown JP, Lang I, et al.: The cyclin-dependent kinase $4 / 6$ inhibitor palbociclib in combination with letrozole versus letrozole alone as first-line treatment of oestrogen receptor-positive, HER2-negative, advanced breast cancer (PALOMA-1/TRIO-18): a randomised phase 2 study. Lancet Oncol 2015;16:25-35.

7 Finn RS, Crown JP, Lang I, et al.: The effect of palbociclib $(\mathrm{P})$ in combination with letrozole $(\mathrm{L})$ on bone metastases in women with ER+/HER2- metastatic breast cancer (MBC): subanalysis from a randomized phase II study. J Clin Oncol 2015;33(suppl):abstr 572.

8 Crown JP, Finn RS, Ettl J, et al.: Efficacy and safety of first-line palbociclib plus letrozole compared with letrozole alone in patients aged $\geq 65$ years with estrogen receptor-positive, HER2-negative advanced breast cancer: a subgroup analysis by age of the PALOMA-1/ TRIO-18 trial. J Clin Oncol 2015;33(suppl):abstr 571.
9 Finn RS, Crown JP, Ettl J, et al.: Clinical efficacy and safety profile of palbociclib $(\mathrm{P})$ in combination with letrozole $(\mathrm{L})$ as first-line treatment in patients (pts) with ER+ and HER2- advanced breast cancer (ABC) who have not received any systemic treatment (ST): a subgroup analysis of PALOMA-1/TRIO-18. J Clin Oncol 2015;33(suppl):abstr 575.

10 Thomssen C, Augustin D, Ettl J, et al.: ABC3 Consensus: assessment by a German group of experts. Breast Care (Basel) 2016;11:61-70.

11 Harbeck N, Iyer S, Turner N, et al.: Quality of life with palbociclib plus fulvestrant in previously treated hormone receptor-positive, HER2-negative metastatic breast cancer: patient-reported outcomes from the PALOMA-3 trial. Ann Oncol 2016;27:1047-1054. 